\title{
Reactivación de Lactobacillus spp. aislados de saliva y alimentos
}

Comparison of substrates for recovery Lactobacillus spp. isolated from saliva and foods
Comparação de substratos para reativar

Lactobacillus spp. isolados de saliva e

alimentos
Facultad de Odontología. Universidad Nacional del Nordeste. Corrientes - Argentina. Biotecnología Microbiana para la Innovación Alimentaria (BiMIA) - (IMIT) Instituto de Modelado e Innovación Tecnológica - CONICET. Docente Auxiliar.

Maria Britos Facultad de Odontología. Universidad Nacional del Nordeste. Corrientes - Argentina. Docente Auxiliar.

Silvia Ortega

Facultad de Odontología. Universidad Nacional del Nordeste. Corrientes - Argentina. Biotecnología Microbiana para la Innovación Alimentaria (BiMIA) - (IMIT) Instituto de Modelado e Innovación Tecnológica - CONICET. Prof. Titular Cátedra de Microbiología e Inmunología.

\section{Olga Vasek}

Facultad de Ciencias Exactas Naturales y Agrimensura. Universidad Nacional del Nordeste. Corrientes - Argentina. Biotecnología Microbiana para la Innovación Alimentaria (BiMIA) - (IMIT) Instituto de Modelado e Innovación Tecnológica. CONICET.

\section{Resumen}

Objetivo: El objetivo del presente estudio fue evaluar la recuperación in vitro del género Lactobacillus de origen bucal y alimentario en medios referenciados como selectivos tales como Rogosa y MRS. Material y Método: se seleccionaron pacientes con caries activas y se recolectaron muestras de saliva que se cultivaron en medio Rogosa; a partir del aislamiento primario las cepas se conservaron en caldo MRS. Se procesaron muestras de leche y agente coagulante artesanal utilizado en la producción de quesos artesanales, los aislamientos primarios se efectuaron en medio Rogosa. Resultados: se aislaron, purificaron y conservaron cepas de Lactobacillus spp. a partir de muestras de saliva y alimentos (leche y agente coagulante artesanal). Conclusión: se demostró que la conservacióna corto y largo plazo del microorganismo en caldo MRS con I5\% de glicerol (crioprotector) a $-20^{\circ} \mathrm{C}$, pudieron, posteriormente ser reactivadas.

\section{Palabras claves}

Bacterias lácticas, microorganismos cariogénicos, conservación de cepas.

\section{Abstract}

Aim: the aim of this study was to evaluate the in vitro recovery of the genus Lactobacillus from oral and food samples on referenced medias selective such as Rogosa. Materials and methods: patients with active caries were selected, and 
saliva samples from the patient self-stimulation were collected by; samples of milk and rennet were processed during the artisanal cheeses production. In Rogosa, development of strains was obtained from a primary isolation of a mixed sample, with subsequent storage in MRS broth. Results: the strains of Lactobacillus spp. were isolated, purified and preserved. Conclusion: it demonstrated that the conservation during short and long times of microorganisms in MRS broth with $15 \%$ glycerol (cryoprotectant) at $-20^{\circ} \mathrm{C}$, could subsequently be reactivated.

\section{Keywords}

Lactic bacteria, cariogenic microorganisms, conservation of strains.

\section{Resumo}

Objetivo: $O$ objetivo do presente trabalho foi avaliar a recuperação em vitro do gênero Lactobacillus de origem bucal e alimentar em meios de cultivos seletivos como Rogosa e MRS. Material e método: selecionaram-se pacientes com cárie ativa e recolheram-se amostras de saliva com estimulaçãodo paciente; processaram-se amostras de leite e o agente coagulante na produção de queijos artesanais. Conseguiu-se o desenvolvimento das cepas, a partir de um isolamento primário de uma amostra mista, com posterior conservação em caldo MRS. Resultado: As cepas de Lactobacillus spp. foram isoladas, purificaradas e conservadas a partir da amostras de saliva e alimentos (leite). Conclusão: Demostrou-se que a conservação a curto ou longo prazo do microorganismo em caldo MRS com $15 \%$ de glicerol (crioprotector) as $-20^{\circ} \mathrm{C}$ puderam, posteriormente, ser reativados.

\section{Palabras chaves}

Bactérias lácticas; microorganismo cariogenicos; preservação de cepas.

\section{Introducción}

En la cavidad bucal existen numerosos microorganismos de diferentes Géneros y especies que habitan las distintas superficies de la misma. Uno de los habitualmente presentes, corresponde al Género Lactobacillus spp. que se aloja en dorso de la lengua, mucosa vestibular, paladar duro, saliva, superficie dentaria formando parte del biofilm y en lesiones de caries activas ${ }^{1,3,7,8}$. Estos lactobacilos se caracterizan por la producción de ácido láctico y forman parte del grupo de bacterias lácticas que, además de ser ubicuos de la cavidad bucal también se encuentran como flora residente en otras partes del cuerpo humano $y$ en diversos alimentos ${ }^{2,11}$.

Los lactobacilos son microorganismos de morfología bacilar, pleomórficos, Gram positivos agrupados en empalizada, anaerobios facultativos o microaerófilos, inmóviles, no esporulados, catalasa negativos ${ }^{6,7,10}$. El género Lactobacillus es descripto como un grupo heterogéneo de "bastones regulares, Gram positivos y no esporulados". Se incluyen dentro del grupo de las bacterias lácticas debido a su capacidad para producir ácido láctico, por lo que desempeñan un rol fundamental en la progresión del proceso de caries $^{6,1,4}$.

Se los clasifica de acuerdo con sus características metabólicas para la asimilación de hidratos de carbono en homofermentativos, que producen ácido láctico como metabolito principal (90\%),heterofermentativos estrictos y heterofermentativos facultativos ${ }^{7}$. La capacidad fermentativa de este microorganismo es su principal factor de virulencia, al generar un ambiente ácido predispone a la desmineralización de tejidos dentales del huésped (esmalte y dentina) produce la destrucción de la pieza dentaria. Fisiológicamente es acidúrico (habita en ambientes con $\mathrm{pHs}$ ácidos y puede seguir produciendo ácido) y acidófilo (habitan en ecosistemas con pHs ácidos). Otras habilidades están relacionadas con su débil pero constante actividad proteolítica, no sintetizan polisacáridos intra $\circ$ extracelulares, no presentanmecanismos de adhesión lo que permite que su establecimiento y residencia sea la anatomía dentaria. $\mathrm{Al}$ ser aerotolerantes desarrollan en atmósferas con $5-10 \%$ de $\mathrm{CO}_{2}$ y requieren de 2 a 3 días de incubación a $35-37^{\circ} \mathrm{C}^{7}$.Se reproducen en medios líquidos y sólidos. Luego del tiempo requerido para el desarrollo y multiplicación, las colonias típicas se presentan de color blanco, forma circular, bordes lisos y regulares, superficie convexa, lisa y brillante, sin pigmentos y un tamaño que varía desde I hasta $5 \mathrm{~mm}$; estas ca- 
racterísticas se observan en medio agarizado ${ }^{7-4}$.

Las especies de lactobacilos que se encuentran, frecuentemente, en cavidad bucal y placa dental son: Lactobacillus (Lb.) rhamnosus, Lb. acidophillus, Lb. salivarius, Lb. gasseri, Lb. crispatus, $L b$. fermentum, Lb. brevis, Lb. Casei y Lb. plantarum ${ }^{7}$.

Los medios de cultivo más utilizados para el desarrollo de Lactobacillus spp. son Rogosa-Mitchell-Wiseman y DeMan-Rogosa y Sharpe.

Rogosa-Mitchell-Wiseman es un medio de cultivo estándar utilizado para el aislamiento de Lactobacillus spp. a partir de la biota humana y alimentos. En su composición, incluye azúcares en proporciones relevantes (glucosa, sacarosa o arabinosa), extracto de levadura y peptona ${ }^{5}$. Se considera un medio selectivo para evitar el crecimiento excesivo de mohos, estreptococos y otros microorganismos. El citrato de amonio, acetato de sodio, ácido acético y el sulfato ferroso actúan como inhibidores de los estreptococos y otros organismos contaminantes. La peptona de caseína, extracto de levadura y sal de amonio, proporcionan la fuente de nitrógeno, Tween 80 suministra los ácidos grasos necesarios para el crecimiento, el manganeso y magnesio actúan como factores de crecimiento y la glucosa es fuente universal de energía y carbono. El fosfato, junto con acetato y ácido acético, estabilizan el $\mathrm{pH}$ en $5,4-5,5^{12}$.

Estudios realizados a partir de muestras de saliva proveniente de 63 individuos, inoculadas sobre medio Rogosa(Merck, Darmstadt, Alemania) mostraron desarrollo de Lactobacillus spp para su posterior recuento ${ }^{9-21}$. El objetivo de este trabajo fue evaluar la recuperación y conservación de Lactobacillus spp. en medios referenciados como selectivos.

\section{Material y Método}

Población: se seleccionaron 20 individuos con caries activas cuyas edades estuvieron comprendidas entre 18 y $2 I$ años. Para el consentimiento informado se utilizó el protocolo aprobado por el Comité de Bioética de la Facultad de Odontología de la UNNE (Resol. I26/II CD). Luego de la firma del consentimiento informado de los pacientes, se realizó el examen bucal y se confeccionó la correspondiente ficha odontológica.
Muestras líquidas. Se procesaron muestras de dos orígenes diferentes: 20 muestras de saliva provenientes de la cavidad bucal que se obtuvieron mediante auto-estimulación por parte del paciente y muestras provenientes de alimentos ( 20 de leche y 20 de agente coagulante artesanal durante la producción de quesos) que se obtuvieron in situ en los establecimientos de manufactura quesera. Para la recolección de ambas, se emplearonrecipientes herméticos estériles que se transportaron al laboratorio en condiciones de refrigeración y se procesaron el mismo día. Para la recuperación de lactobacilos, se utilizó el medio Rogosa (BiokarDiagnostics, Beauvais, Francia), preparado según las indicaciones del fabricante y luego se adicionó ácido acético para lograr el pH de 5,5. Las muestras se inocularon mediante la metodología de siembra en superficie sobre el medio solidificado en cajas de Petri; se empleó $0, \mathrm{I} \mathrm{mL}$ de saliva en una dilución de I/5 como inóculo y se distribuyó con espátula de Drigalsky; las cajas de Petri se incubaron durante $48 \mathrm{~h}$ a $37^{\circ} \mathrm{C}$ en atmósfera de $\mathrm{CO}_{2}$. Las colonias que presentaron caracteres típicos del género Lactobacillus spp., color blanco, forma circular, bordes lisos y regulares, superficie convexa, y tamaño entre I y $5 \mathrm{~mm}$, y su microscopía correspondió a bacilos de extremo redondeado, se seleccionaron para su purificación y conservación. Se realizaron pasajes sucesivos desde un medio sólido (Rogosa) a líquido (caldo De Man-Rogosa y Sharpe, MRS-Lab. Britania-Argentina) incubando a $37{ }^{\circ} \mathrm{C}$ durante 24 h. La pureza de las cepas se comprobó por microscopía y el contenido se fraccionó en tubos Eppendorf empleando glicerol (I5\%) como crio-protector para su conservación a $-20^{\circ} \mathrm{C}$.

\section{Resultados}

Del aislamiento primario de las 20 muestras de saliva procesadas se obtuvo desarrollo de microorganismos con características macromorfológicas típicas (Fig. I) en el total de las muestras ( $100 \%$ de recuperación). Todos los aislados fueron catalasa (-) y, a la tinción de Gram mostraron ser bacilos Gram +, cortos, de extremo redondeado y pleomórficos (Fig. 2) que se clasificaron como Lactobacillus spp. 
De las 20 muestras de leche procesadas, se logró la recuperación de lactobacilos solo en 2 de ellas ( $10 \%$ de recuperación) y de las 20 muestras de agente coagulante, se recuperaron lactobacilos en el $100 \%$ de ellas, presentando una carga microbiana promedio de 8,0.107 unidades formadoras de colonias $/ \mathrm{mL}$. Las cepas obtenidas se conservaron por congelación en medio líquido MRS con adición de glicerol al 15\% como crioprotector durante I5 días, 6 meses y I año. Luego de transcurridos los mencionados tiempos, las cepas se reactivaron logrando desarrollo en aquella almacenadas durante 15 días y 6 meses de ambos orígenes, oral y alimentario (Fig. 3). Las cepas de origen alimentario conservadas durante I año, se recuperaron exitosamente, no así las cepas de origen humano.

\section{Discusión}

Los lactobacilos son un grupo de microorganismos que habitan el cuerpo humano y forman parte de la biota de algunos alimentos. Por sus características metabólicas, algunas especies son utilizadas como cultivos iniciadores o como parte de ellos, para la manufactura de diferentes alimentos fermentados, como quesos artesanales ${ }^{13}$. Sus metabolitos de origen peptídico ejercen actividad antagónica frente al crecimiento de diferentes especies bacterianas ${ }^{14}, 15$, por lo cual poseen gran interés industrial como bio-preservantes inocuos para la salud humana y fundamentanla importancia de su cultivo y preservación. En el área de la salud odontológica, por ser habitantes de la cavidad bucal, se considera importante su recuento para determinar el riesgo microbiológico de actividad de caries de los sujetos que padecen esta enfermedad ${ }^{2 !}$.

La preservación y mantenimiento de las cepas de origen bucal cultivadas y conservadas a bajas temperaturas, con la adición de crioprotectores es importante para garantizar su pureza, sin variaciones ni mutaciones, es decir, conservar el estado fisiológico, metabólico y genético originales presentes en el microorganismo lo cual permite utilizar las cepas aisladas e identificadas para diferentes propósitos: facilitar el proceso enseñanza - aprendizaje en la formación de recursos humanos y docencia de grado y posgrado, en la elaboración o biopreservación de variados pro-

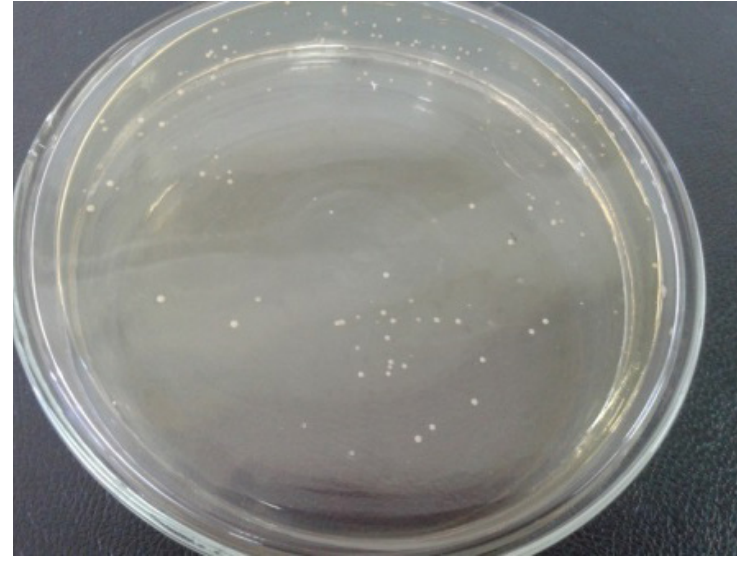

Figura I. Aislamiento primario de Lactobacillus.

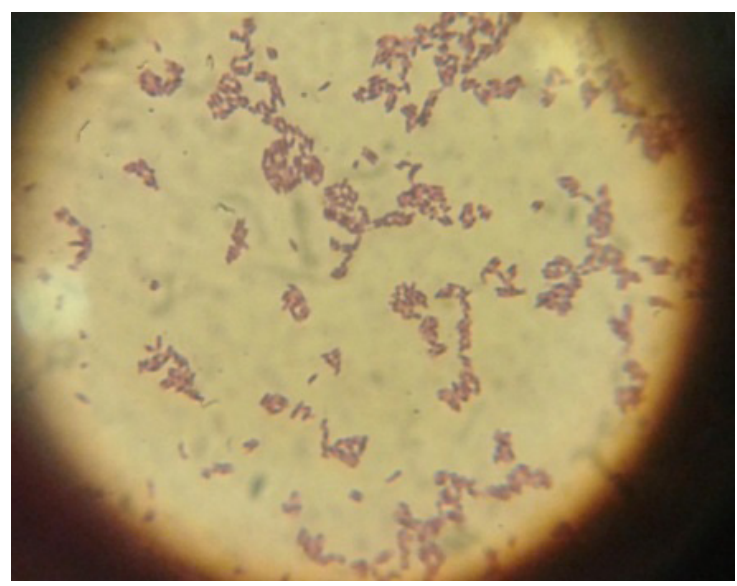

Figura 2. Tinción de Gram (+).

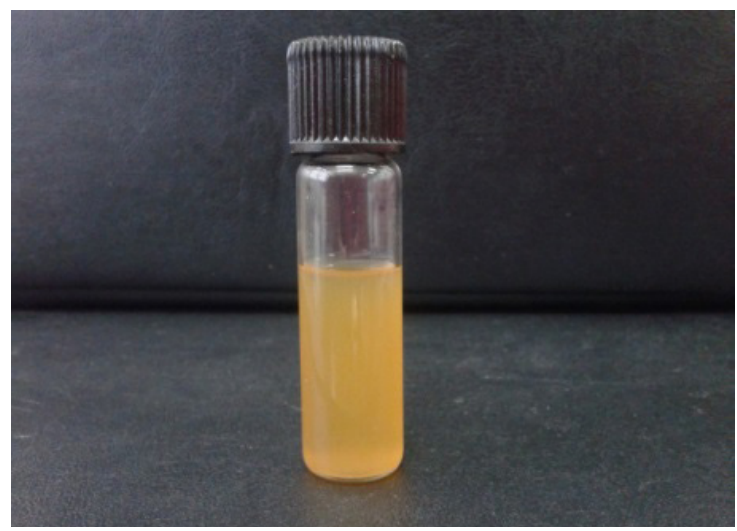

Figura 3. Reactivación de cepa en mediode cultivo líquido. 
REVISTA FACULTAD DE ODONTOLOGÍA

ISSN No 1668-7280 - Vol. IX No 1 - 2016

11
INVESTIGACIÓN

Reactivación de Lactobacillus spp. aislados

de saliva y alimentos ductos en la industria y en evaluaciones diagnósticas genéticas, epidemiológicas, inmunológicas y patológicas $^{20}$.

Alvarado Rivas y col., emplearon agar MRS para el aislamiento primario de lactobacilos a partir de quesos artesanales. Seleccionaron las colonias más representativas, las inocularon en igual medio líquido y las incubaron en condiciones de microaerofilia durante $48 \mathrm{~h}$ a $37^{\circ} \mathrm{C}$. Para la conservación de estas cepas crecidas adicionaron al caldo MRS, 30 \% de glicerol como crioprotectory para su recuperación utilizaron este mismo medio ${ }^{16}$.

Ortega y col., realizaron el aislamiento de Lactobacillus spp. en medio Rogosa para realizar el recuento de unidades formadores de colonias por mililitro de saliva (ufc/mL), con el objetivo de determinar la actividad de caries en un grupo de pacientes con edades entre 8 y 15 años que presentaban diabetes tipo $\mathrm{I}^{17}$.

Sánchez y col., realizaron el aislamiento de cepas de Lactobacillus spp. a partir de muestras vaginales en caldo y agar MRS, utilizándolos a su vez como medios de conservación a corto plazo mantenidos a $4-8{ }^{\circ} \mathrm{C}$ realizando repiques mensuales. Para la conservación a largo plazo emplearon caldo MRS con la adición de glicerol (30\%). Lograron recuperar las cepas conservadas en el mismo medio liquido con la finalidad de evaluar la actividad antagónica in vitro frente a patógenos clínicos tales como Candida albicans y Escherichia coli' ${ }^{18}$.

En función de los resultados que se presentan, se establece que la utilización del medio Rogosa otorga seguridad para el aislamiento selectivo primario de muestras líquidas de origen bucal exitosamente ( $100 \%$ de recuperación total), en concordancia con los autores antes mencionados. Sin embargo, no es el mejor medio para la recuperación de Lactobacillus spp. a partir de muestras líquidas de origen lácteo $(55 \%$ de recuperación total), para lo cual se recomienda habitualmente el empleo del medio MRS ${ }^{19}$. El medio líquido de igual composición (MRS) permitió la purificación y conservación de cepas de lactobacilos de ambos orígenes.

\section{Conclusiones}

El análisis de los resultados obtenidos permite enunciar que:

- El agar Rogosa proporcionó las condiciones para el aislamiento selectivo de lactobacilos a partir de muestras líquidas de origen bucal.

- El medio líquido (MRS) utilizado para la reproducción y purificación de Lactobacillus spp. obtenidos a partir de muestras líquidas de la cavidad bucal y de alimentos de origen lácteo, permitió, además, ser empleado para la conservación por congelación de las cepas aisladas durante un tiempo de almacenamiento de 6 meses para los aislados bucales y I año para los de origen lácteo, bajo las condiciones especificadas.

\section{Aprobación Ética y Consentimiento/ Asentimiento}

Consentimiento informado aprobado por el Comité de Bioética de la Facultad de Odontología de la UNNE (Resol. I26/I I CD).

\section{Conflictos de intereses / Fuentes de fi- nanciamiento}

Fuente de financiamiento: Secretaria General de Ciencia y Técnica - Universidad Nacional del Nordeste. 


\section{Referencias}

I. Ahumada MC, Bru E, Colloca ME, López ME, NaderMacías ME. Evaluation and comparison of lactobaciIli characteristics in the mouths of patients with or without cavities [En línea]. J. Oral Science. 2003. 45 (I). I-2. Disponible en: https://www.jstage.jst.go.jp/ article/josnusd 1998/45/I/45_I_I/_pdf

2. Alonso Buriti FC, IsaySaad SM. Bactérias do grupo Lactobacillus casei: caracterização, viabilidade comoprobióticos em alimentos e sua importancia para a saúde humana [Em linea]. Arch Latinoam de Nutricion. 2007. 57(4) 373-374. Disponible en: http://www. scielo.org.ve/pdf/alan/v57n4/art 10.pdf

3. Badet C, Thebaud NB. Ecology of Lactobacilli in the Oral Cavity: A Review of Literature. The Open Microbiology Journal [En linea]2008.2, 38-48. http:// www.ncbi.nlm.nih.gov/pmc/articles/PMC2593047/pdf/ TOMICROJ-2-38.pdf

4. Hammes W, Weiss N, Holzapfel W. The genera Lactobacillus and Carnobacterium. En: Ballows A, Trüper $H$, Harder W, Dworkin M, SchleiferKH. The Procariotes. $2^{\mathrm{a}}$ ed. Nueva York, Springer-Verlag. 1992.1535-1593

5. Rogosa M, Mitchell JA, WisemanRF.A selective medium for the isolation and enumeration of oral and fecal lactobacilli. J of Bacteriol.[en linea]. I95।.62:I.I32-I33. Disponible en:http://jb.asm.org/content/62/I//32.full. pdf+html

6. Figueroa GM, Alonso G, Acevedo AM. Microorganismos presentes en las diferentes etapas de la progresión de la lesión de caries dental. Acta Odontológica Venezolana.[en línea]. Vol 47: I. 2009. 4-5. Disponible en: http://www.actaodontologica.com/ediciones/2009/I/pdf/microorganismos_progresion_lesion_ caries_dental.pdf

7. Liebana Ureña J. Microbiología Oral. $2^{2}$ Ed. Edit. Interamericana. |985. 350-351

8. Negroni M. Microbiología Estomatológica. Fundamentos y guía práctica.2ªd. Edit. Panamericana. 1999. 239.

9. Giacaman RA, Muñoz-Sandoval C, Bravo González E, Farfán-Cerda P. Cuantificación de bacterias relacionadas con la caries dental en saliva de adultos y adultos mayores. Rev Clin Periodoncia Implantol Rehabil Oral 2013; 6 (2): 7I-73 [en línea]. Disponible en: http:// scielo-test.scielo.cl/pdf/piro/v6n2/art04.pdf

10. Pelinescu D R, Sasarman E, chifiriuc M C, Stoica I, Nohit A M, Avram I, et al. Isolation and identification of some Lactobacillus and Enterococcus strains by a polyphasic taxonomical approach. Romanian Biotechnological Letters 2009; I4 (2): 4225-4233. Disponible en: http://www.rombio.eu/rb/2voll4/cnt/Lucr-3.pdf

II. Ramirez Ramirez JC, Ulloa PR, Velasquez Gonzalez MY, Ulloa JA, Romero FA. Bacterias lácticas: importancia en alimentos y sus efectos en la salud. Revista Fuente. 20II; 7. Disponible en: http://fuente.uan.edu. $\mathrm{mx} /$ publicaciones/03-07/I.pdf
12. Zimbro JM, Power DA, Miller SM, Wilson GE, Johnson JA. Difco\& BBL Manual of Microbiological Culture Media. Sparks, Maryland 2009; 2a 289-290. Disponible en: https://www.bd.com/ds/technicalCenter/ misc/difcobblmanual_2nded_lowres.pdf

13. Vasek O, Mazza S, de Giori GS. Physico-Chemical and microbiological evaluation of Corrientes Artisanal Cheese during ripening. Food Sci Technol 2013; 33 (I): I5I-I60.

14. Zoumpopoulou G, Pepelassi E, Papaioannou W et al. Incidence of Bacteriocins Produced by Food-Related Lactic Acid Bacteria Active towards Oral Pathogens. Int J Mol Sci 20I3; 14(3): 4640-4654. Disponible en: http://www.ncbi.nlm.nih.gov/pmc/articles/ PMC3634443/

15. Sin C, Vasek O, Ortega S. Metabolitos de bacterias lácticas antagónicos al crecimiento de microorganismos cariogénicos. Rev. Asoc. Arg. Investig odontol 20I5; 103 (I): 4-8.

16. Alvarado Rivas C, Chacón Rueda Z, Otoniel Rojas J y col. Aislamiento, Identificación y Caracterización de Bacterias Ácido Lácticas de Venezolano Ahumado Andino Artesanal. Su Uso Como Cultivo Iniciador [En Linea]. Rev Cient (Maracaibo) 2007; I7(3): 30I-306. Disponible en: http://www.scielo.org.ve/pdf/rc/v17n3/ art I4.pdf

17. Ortega, S, Rodriguez V, Paniagua S. Indice CPOD, niveles de Streptococos mutans, Lactobacilos e inmunoglobulina $A$ en pacientes diabéticos infantojuveniles en el Hospital Pediátrico de la Ciudad de Corrientes. Revista de la Facultad de Odontología de la U.N.N.E. 2009; 2: 2.

18. Sánchez L, Vichi J, Llanes M y col. Aislamiento y caracterización in vitro de cepas de Lactobacillus spp. como candidato a probióticas [En línea]. Rev Salud Anim 20I I; 33 (3). Disponible en: http://scielo.sld.cu/pdf/ $\mathrm{rsa} / \mathrm{v33n3/rsa033II.pdf}$

19. De Man JC, Rogosa M, Sharpe ME. 1960. En: Harrigan W. F., and M. E. McCance. Laboratory methods in food and dairy microbiology. Academic Press, London. 1976. p.:205.

20. Gamboa Jaimes FO. Estrategias y procedimientos para la conservación de bacterias de importancia en la cavidad bucal. Rev Asoc Odontol Colomb 2008; 7I (224): 9-15. Disponible en: https://issuu.com/fodontocol/ docs/foc_no_224_I_.

21. Giacaman RA, Muñoz-Sandoval C, Bravo González E, Farfán-Cerda P. Quantification of caries-associated bacteria from saliva of adults and older adults. Rev Clin Periodoncia Implantol Rehabil Oral 2013; .Vol. 6(2); 7 II-74. 